\title{
Kinetic Studies on Lipase-catalyzed Transesterification of Phosphatidylcholine with $\alpha$-linolenic Acid Ethyl Ester
}

\author{
Junmin Du (Corresponding author) \\ State Key Laboratory of Coal Conversion \\ Institute of Coal Chemistry Chinese Academy Sciences, Taiyuan, China \\ $\&$ \\ Graduate University of the Chinese Academy of Sciences, Beijing, China \\ Tel: 83-351-408-4708Ｅ-mail: dujm@sxicc.ac.cn \\ Dong Wu \& Xianglin Hou \\ Institute of Coal Chemistry Chinese Academy Sciences, Taiyuan, China
}

Cuiping Feng

College of Food Science and Technology, Shanxi Agricultural University, Taigu, China

The research is financed by the Chinese Academy of Sciences. No. 06SC7413B1 (Sponsoring information)

\begin{abstract}
The kinetics of the transesterification of phosphatidylcholine (PC) with $\alpha$-linolenic acid ethyl ester (ALAEE) catalyzed by immobilized lipase in hexane was studied. The reaction proceeded via a Ping-Ping Bi-Bi mechanism without inhibition by both the substrates at various concentrations tested. A reaction kinetic model was proposed with the experimental data. The kinetic constants of the model were determined at $45^{\circ} \mathrm{C}$, which were $1.2 \times 10^{-2} \mu \mathrm{mol} . \mathrm{min}^{-1} . \mathrm{mg}^{-1}, 64.1 \mathrm{mM}$ and $282.8 \mathrm{mM}$ for Vmax, KmALA and KmPC, respectively.
\end{abstract}

Keywords: Phosphatidylcholine, A-linolenic acid, Kinetic study, Ping-Ping Bi-Bi mechanism

\section{Introduction}

The phospholipids (PL) form of n-3 polyunsaturated fatty acids (n-3 PUFA) is considered to be nutritionally more favorable than other fatty acid derivatives, such as methyl or ethyl esters even triglycerides (TG). It is because that phospholipids form not only provides high digestion of fatty acid to cell (Dominique et al., 1999), but also has considerable special functions. For example, DHA-containing PL can promote cell differentiation in leukemia (Hosokawa et al., 1999), enhance survivals of tumor-bearing mice (Jenski et al., 1995), and prevent cerebral apoplexy (Eibl et al., 1988). After hydrolysis, phospholipids with PUFA can be decomposed into PUFA and PL, which can act on cell in concurrence and show an increased double effect on human body.

A-linolenic acid (ALA) belongs to n-3 polyunsaturated fatty acids (PUFA). It is the precursor of EPA and DHA and also shows the similar high bioactivity in vivo. Compared with PL including EPA and DHA, an advantage of the synthesis of type- ALA phospholipids with PUFA is no residue of cholesterol in the PL productions. Since PUFA donor ALA is from plant oil (linseed oil) whereas EPA and DHA are from fish oil which has a lot of worries of cholesterol from animal. Therefore, the synthesis of PL of ALA type is very significant to new PL with PUFA.

The simplest and most direct route for the synthesis of ALA type PL is the transesterifiction between PL and ALA catalyzed with a lipase. Lipases offer high catalytic efficiency, specificity and selectivity by incorporation of the required acyl group into a specific position of the native PL. Some studies (Chojnacka et al., 2009; Junmin et al., 2009) have focused on the reaction conditions including temperature, time, water content, and substrate concentration rate. However, little information has been reported on the kinetics of the lipase-catalyzed transesterifiction between PL and ALA and the rate equations for the reaction mechanism involving magnitude of constants, especially on using ALAEE as acyl donor by gas chromatography, were thus absent. Kinetic studies are the basis for elucidating the transesterification mechanism and designing a bioreactor (Garcia et al., 1999). 
The synthesis of type-ALA phospholipids with PUFA by lipase-catalyzed transesterification in hexane can provide more information about these 2 aims. We studied the kinetics on lipase-catalyzed transesterification of phosphatidylcholine (PC) from egg with $\alpha$-linolenic acid ethyl ester (ALAEE) by gas chromatography.

\section{Experimental}

\subsection{Materials}

Egg-yolk PC (purity $98.5 \%$, average molecular weight $779 \mathrm{~g} / \mathrm{mol}$ ) was self-made by supercritical $\mathrm{CO}_{2}$ extraction and purification with column chromatography. $\alpha$-Linolenic acid methyl ester (C18:3, purity 98.5\%) was purchased from Sigma-Aldrich (Shanghai, China). Immobilized lipase Lipozyme TL IM, an sn-1, 3 specific lipase from Thermomyces lanuginosa, was donated by Novoymes (Shenyang, China). Solvents used and all other chemicals were of analytical grade and obtained commercially.

\subsection{Enzyme reactions}

A typical experiment was run at $45{ }^{\circ} \mathrm{C}$ in a $5 \mathrm{ml}$ container with magnetic stirring at $100 \mathrm{rpm} .100 \mathrm{mg}$ of immobilized enzyme was added to a total of $4 \mathrm{ml}$ hexane reaction solution containing PC and ALAEE. After reactions, the samples were centrifuged and the supernatants were collected. All samples were stored at $-20{ }^{\circ} \mathrm{C}$ prior to analysis of GC. The initial reaction rate was determined after 60 min reaction and it was represented as the amount of ALAEE decreased per unit time within the limits of less than $5 \%$ of transesterification ( for considering no product inhibition).

\subsection{Water content control}

Prior to reaction, the PC and ALAEE in the hexane were dehydrated over 4A molecular sievers over $4 \mathrm{~h}$ and filtrated out. The immobilized enzyme was dehydrated under vacuum until the weight constant.0.0, 2.5, 5.0, 7.5, $10,12.5,15,17.5$ and $20 \%$ water content (by the enzyme weight) was obtained with addition of a known quantity of distilled water to the dried enzyme. The wet enzymes were stored at $4{ }^{\circ} \mathrm{C}$ for one week. Water content in the media was analyzed on a $684 \mathrm{KF}$ Coulometer.

\subsection{Analysis of fatty acids by gas chromatography}

Fatty acid ethyl or methyl esters were analyzed on a Shimadzu gas chromatograph GC-14B with flame-ionization detector (Shimadzu, Kyoto, Japan). The glass column packed with 20\% DEGS on celite (3mm i.d. $\times 2 \mathrm{~m}$ length) was employed; the column temperature was $190{ }^{\circ} \mathrm{C}$ and the detector temperature was $250{ }^{\circ} \mathrm{C}$. The n-eicosane was added in reaction solution prior to reaction as internal standard to calculate the amount of various fatty acid esters in the reaction system.

\section{Results and Discussion}

The transesterification of PC and ALAEE for ALA containing PC production was shown in Scheme 1(Fig.1). After reaction, the ALAEE content would decrease while the content of palmitic acid and stearic acid ethyl esters (PAEE and STEE, abbreviated respectively and can be lumped as a single constituent for comparison) formed would increase in the solution.

\subsection{Effect of rotation speed and enzyme concentration}

In this study, a $100 \mathrm{rpm}$ of stirring speed was adopted and the mass transfer limitations could be neglected after investigation of rotating speed $(60-300 \mathrm{rpm})$. When increasing the enzyme concentration, the initial reaction rate was also increased as shown in Fig.2. It was verified that the reaction was controlled by kinetics.

\subsection{Effect of water content}

Magnus Harrod et al. (1995) observed that there was no reaction at low water activity for synthesis of PC. Similar results were observed in our test. But when water content of enzyme increased, the initial rate began to increase (Fig.3), indicating that water is essential for maintaining lipase activity. Vikbjerg et al. (2007) considered that water addition was the most significant factor on the PLA2 catalyzed acidolysis reactions in terms of incorporation and recovery. One reason was that water enhanced the molecular flexibility favorable to reaction in hexane. Another reason was that the reaction intermediates must be hydrolyzed first through the nucleophilic attack by water. Miller et al. (1991) and Reyes et al. (1994) have probed that water was needed to rupture the native ester bonds by the first step of hydrolysis reaction in the transesterification of triglycerides. In the case of PC and ALAEE, Dry lipase indicated that no water was available for rupturing the substrate ester bonds and reaction did not proceed although both PC and ALAEE can be used as nucleophile directly for transesterification. When the water content of enzyme was varied from 0.0 to $7.5 \%$ (by weight), the initial rate of consumption of ALAEE increased fast and after that it increased continuously but slowly. This seems be 
explained that this lipase tends to favor the more water content to the transesterification reaction. However, the lumped values of PAEE and STEE were not in good agreement with the concentration of ALAEE decreased under higher water content (more than 7.5\%) with further investigation of product ethyl esters (Fig.3). The discrepancies between them became larger and larger as the initial water content increased in the enzyme. The lower hydrolysis rate of PC compared to ALAEE may be one of the main reasons, suggesting that the hydrolysis of PC for intermediate LPC can be rate-limiting step to the overall transesterification. Low water activity (making it possible to keep competing hydrolysis low) is needed in the lipase-catalysed transesterification (Lin et al. 2002). When the water content of enzyme reached at $5.0 \%$, the discrepancy resulting from hydrolysis became the smallest and the hydrolysis side reaction can be negligible. The hydrolysis of ALAEE was suppressed to match that of PC effectively and the one-step transesterification of PC can proceed truly.

\subsection{Effect of reaction time}

In the most appropriate water content of 5.0\%, there was a slow increase in the initial rate of ALAEE in the first minutes of reaction due to the absence of intermediates, indicating that the reaction needs a long time to react (Fig.4). However, after a long time of incubation, the reaction proceeds fast. It should be the accumulated intermediates that resulted in the fast reaction velocity. After about $3 \mathrm{~h}$, the reaction reached to equilibrium. No significant decrease in the initial rate during the later period of reaction, indicating that no product inhibition occurred. In the initial reaction time, there was a decrease in the water content in the first hours of reaction, indicating that water was consumed for hydrolysis (Fig.4). The consumed water was also released by the re-esterification followed product. It neither increased nor decreased in the overall reaction process. To obtain precise reaction rate, $60 \mathrm{~min}$ of reaction time was suggested good for determining of initial rate taking into consideration that the substrates was converted less than 5\% as well as LPC present at low concentrations.

\subsection{Effect of substrate concentration}

We investigated the effect of substrate concentration on the initial reaction in appropriate water content of 5.0\% and reaction time of $60 \mathrm{~min}$. The results are shown in Fig.5. It is obvious that when one of the substrate concentrations is kept constant, the initial reaction rate increased with increasing concentration of another substrate. Also, no evidence for the phenomenon of substrate inhibition can be seen. Alcohol always results in inhibition as alcohol substrate molecule can act as a dead-end inhibitor in the esterification reaction (Chulalaksananukul et al., 1990; Janssen et al., 1999), but alcohol in ALAEE has been esterified with long chain fatty acid, which contributes to certain non-polar effect on protecting enzyme structure from losing water in the present reaction. Appropriate water content (5.0\% based on enzyme) has a key effect on the reaction (Reyes et al., 1994). Water increases enzyme molecular flexibility avoiding exceeding hydrolysis of esters and improves the reaction rate and provides good condition for the transesterification without inhibition of substrates and products.

\subsection{Kinetic model based on initial rates}

Experimental data in Fig.5 was converted into double-reciprocal plot of initial rate of transesterification at varying contents. The plot of 1/V for 1/ALA presents a group of parallel model seen in Fig.6. This indicated that the reaction was in good agreement with Ping-Pong mechanism, which is characterized by parallel lines in the Lineweaver-Burk (double reciprocal) representation at concentrations in which there is no inhibition, as it happens in the present system.

Transesterification of PC with ALA ethyl ester is a two substrate reaction involving two steps. The scheme of the reaction steps was followed as Fig.7. In the first step, the acyl of either PC or ALA ethyl ester binds the free enzyme (E) forming a noncovalent enzyme-ester complex which is transformed to an enzyme-acyl-alcohol intermediate, which is further hydrolyzed as new enzyme-alcohol intermediate $(\mathrm{F})$ with the concomitant release of the first product, either sn-1 positional fatty acids of PC or ALA. In the second step, the new enzyme-alcohol intermediate is esterified with another fatty acid, as new enzyme alcohol-acid would be first formed and then a molecule of water is released and esterification is performed. After that the enzyme-ester intermediate is formed and further isomerized to the original enzyme and the release of the second product, either new PC or new ethyl ester.

According to the mechanism above, the rate equation for the mechanism (Segel, 1975) is given by:

$$
V=\frac{V \max \left[\mathrm{C}_{\mathrm{PC}}\right]\left[\mathrm{C}_{\mathrm{ALAEE}}\right]}{K_{\mathrm{mALA}}\left[\mathrm{C}_{\mathrm{PC}}\right]+K_{\mathrm{mPC}}\left[\mathrm{C}_{\mathrm{ALAEE}}\right]+\left[\mathrm{C}_{\mathrm{PC}}\right]\left[\mathrm{C}_{\mathrm{ALAEE}}\right]}
$$


Where $\mathrm{V}$ is the initial reaction rate; $\left[\mathrm{C}_{\mathrm{PC}}\right]$ and $\left[\mathrm{C}_{\mathrm{ALAEE}}\right]$ are the concentrations of $\mathrm{PC}$ and ALA ethyl ester, respectively; $V_{\max }$ is the maximum velocity or limiting rate; $\mathrm{K}_{\mathrm{mPC}}$ and $\mathrm{K}_{\mathrm{mALAEE}}$ are the Michaelis constants for PC and ALA ethyl ester, respectively.

Intercepts of straight lines in the primary plots in Fig.6 were plotted for the reciprocal of contents (Fig.8.). From Fig. 6 and Fig. 8 , the kinetic constants were obtained. That is: $\mathrm{V}_{\max }=1.2 \times 10^{-2} \mu$ mol. $\mathrm{min}^{-1} \cdot \mathrm{mg}^{-1}$; $\mathrm{K}_{\text {mALAEE }}=64.1 \mu$ $\mathrm{mol} / \mathrm{mL} ; \mathrm{K}_{\mathrm{mPC}}=282.8 \mu \mathrm{mol} / \mathrm{mL}$. The equation follows as below:

\subsection{Validating of model}

$$
V=\frac{1.2 \times 10^{-2}\left[\mathrm{C}_{\mathrm{PC}}\right]\left[\mathrm{C}_{\mathrm{ALAEE}}\right]}{64.1\left[\mathrm{C}_{\mathrm{PC}}\right]+282.8\left[\mathrm{C}_{\mathrm{ALAEE}}\right]+\left[\mathrm{C}_{\mathrm{PC}}\right]\left[\mathrm{C}_{\mathrm{ALAEE}}\right]}
$$

With the kinetic rate expression available, it is also possible to model the initial reaction rates using the equation (2) and to compare these with the experimentally observed values. A plot of the experimental rate values versus the calculated rate values give a straight-line which almost passes through the experimental points (Fig.9), showing that the experimental rate data match the values calculated by the model well.

As can be seen from Fig.9 for the lipase, PC has a lower affinity than ALAEE as $\mathrm{K}_{\mathrm{PC}}$ is higher than ALAEE's. The higher $k_{\mathrm{PC}}$ value may be caused from its bulky and polar molecular characterization and this can lead to the time-consuming and low efficiency in the modificaition of PC. From an applied point of view, a lipase bearing similar $\mathrm{K}$ value for the two substrates will enhance reaction rate efficiently since the transesterification is run synchronously. If some of new measures for reaction, for example, new lipases, can be taken, the transesterification will be enhanced rapidly and greatly. Nevertheless, the result obtained was consistent with the effect of higher water content above where the hydrolysis of PC for intermediate LPC can be rate-limiting step to the overall transesterification. Perhaps, other reaction details such as the re-esterification LPC needs further investigation, however, this model is able to account for the effects of the concentration of the chemical species participating in transesterification throughout the entire reaction and it also provides an easy model of study for the lipase-catalyzed synthesis of ALA type phospholipids with PUFA.

\section{In conclusion}

The kinetics of the transesterification of phosphatidylcholine with a-linolenic acid ethyl ester catalyzed by immobilized lipase in hexane was studied. Under the appropriate conditions $(5.0 \%$ water content of enzyme and $60 \mathrm{~min}$ of reaction time), the reaction follows Michalis-Menton equation and a Ping-Ping Bi-Bi mechanism without inhibition by the both substrates at all initial concentrations tested. Water has a key effect on enzyme activity and the hydrolysis of PC for intermediate LPC can be rate-limiting step to the overall transesterification, suggesting that new methods or lipases are needed for improving reaction rate. A simple kinetic model of reaction was proposed and fitted well with the experimental data. The kinetic constants of the model were $1.2 \times$ $10^{-2} \mu \mathrm{mol}_{\mathrm{min}} \mathrm{m}^{-1} . \mathrm{mg}^{-1}, 64.1 \mathrm{mM}$ and $282.8 \mathrm{mM}$ for $\mathrm{V}_{\mathrm{max}}, \mathrm{Km}_{\mathrm{ALA}}$ and $\mathrm{K}_{\mathrm{mPC}}$, respectively.

\section{Acknowledgments}

Financial support from the Knowledge Innovation Programs of the Chinese Academy of Sciences (06SC7413B1) is gratefully acknowledged.

\section{References}

Chojnacka A., Gladkowski W., Kielbowicz G., Wawrzenczyk C. (2009). Enzymatic enrichment of egg-yolk phosphatidylcholine with a-linolenic acid. Biotechnol. Lett., 31:705-709.

Chulalaksananukul W., Condoret J.S., Delorme P. and Willemot R.M. (1990). Kinetic study of esterification by immobilized lipase in n-hexane. FEBS.Lett., 276 (12)181-184.

Dominique L D, Christlane P, Martinel L, et al. (1999). Blood compartmental metabolism of docosahexaenoic acid (DHA) in humans after ingestion of a single dose of [13C] DHA in phosphatidylcholine. J. Lipid Res., 40: $1867-1874$.

Eibl H, Unger C. (1988). Phospholipids-selective drugs in cancer therapy. Proc. Soc. Exp. Biol. Med., 29:358.

Garcia, T., Sanchez, N., Martinez, J., Aracil, J. (1999). Enzymatic synthesis of fatty acid esters. Part I. Kinetic approach. Enzyme Microbiol. Technol., 25, 584-590.

Hosokawa M, Minami K, Kohno H, Tanaka Y, Hibino H. (1999). Differentiation- and apoptosis-inducing activities of phospholipids containing docosahexaenoic acid for mouse myeloid leukemia M1cells. Fisheries Sci., 65(5): 798-799. 
Janssen AEM, Sjursnes BJ, Vakurov AV, Halling P. (1999). Kinetics of lipase-catalyzed esterification in organic media: correct model and solvent effects on parameters. Enzyme Microb Technol., 24: 463-70.

Jenski LJ, Zerouga M, Stillwell W. (1995). O-mega3 fatty acids in cancer therapies. Proc. Soc. Exp. Biol. Med., 210: $227-233$.

Junmin Du, Dong Wu, Cuiping Feng, Xianglin Hou. (2009). Enzymatical synthesis (a -LNA) of phosphatidylcholine containing polyunsaturated fatty Acids. J.Mol.Catal. (China), 23(4):372-376.

Lin Ma, Mattias Persson, Patrick Adlercreutz. (2002). Water activity dependence of lipase catalysis in organic media explains successful transesterification reactions. Enzyme Microbiol. Technol., 31:1024-1029.

Magnus Harrod and Inger Elfman. (1995). Enzymatic synthesis of phosphatidylcholine with fatty acids, isooctane, carbon dioxide, and propane as solvents J. Am. Oil Chem. Soc., 72:641-646.

Miller Douglas A., Prausnitz John M. and Blanch. Harvey W. (1991). Kinetics of lipase-catalysed interesterification of triglycerides in cyclohexane. Enzyme Micro. Technol., 13(2):98-103.

Reyes Hector R., Hill Charles G., Jr. (1994). Kinetic modeling of interesterification reactions catalyzed by immobilized lipase. Biotechnol. Bioeng., 43: 171-182.

Segel, IH. (1975). Enzyme Kinetics: Behaviour and analysis of rapid equilibrium and steady-state enzyme systems. New York. John Wiley and Sons, pp: 606-612.

Vikbjerg A. F., Mu H., Xu X. (2007). Synthesis of structured phospholipids by immobilized phospholipase A2 catalyzed acidolysis. J. Biotechnol., 128: 545-554.
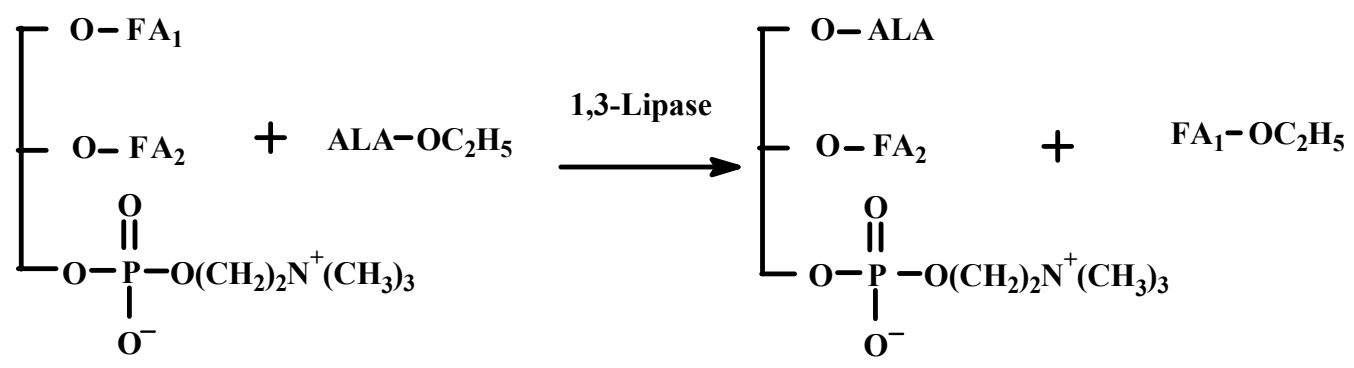

$\mathbf{F A}=$ Fatty acid residual, $\mathbf{A L A}=\mathrm{A}$-linolenic acid residual

Figure 1. The scheme of the reaction catalyzed by a lipase

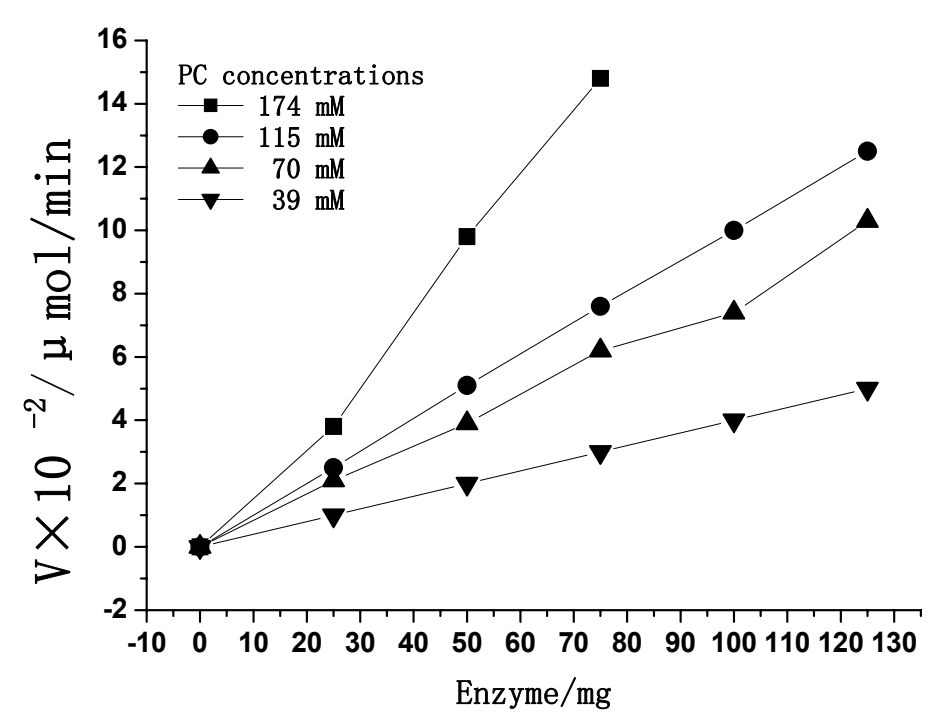

Figure 2. Effect of enzyme content on the initial reaction rate 


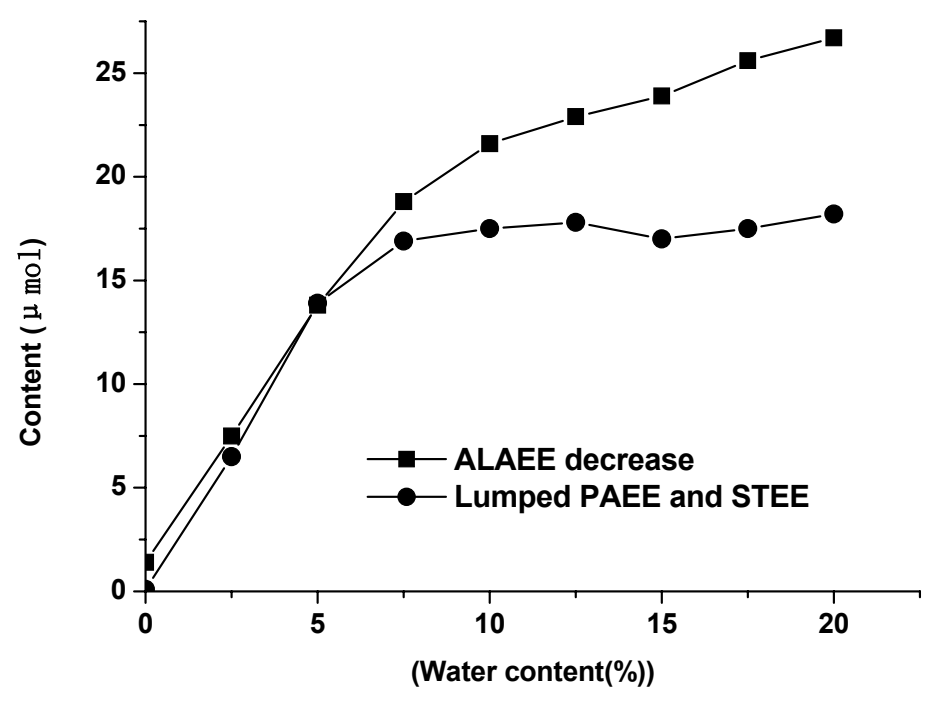

Figure 3. Contents of the constituents in the solution as a function of water content. Reaction condition: PC (90.5 $\mathrm{mg})$, ALAEE (120.0 mg), reaction time (60 min), lipase (100 mg)

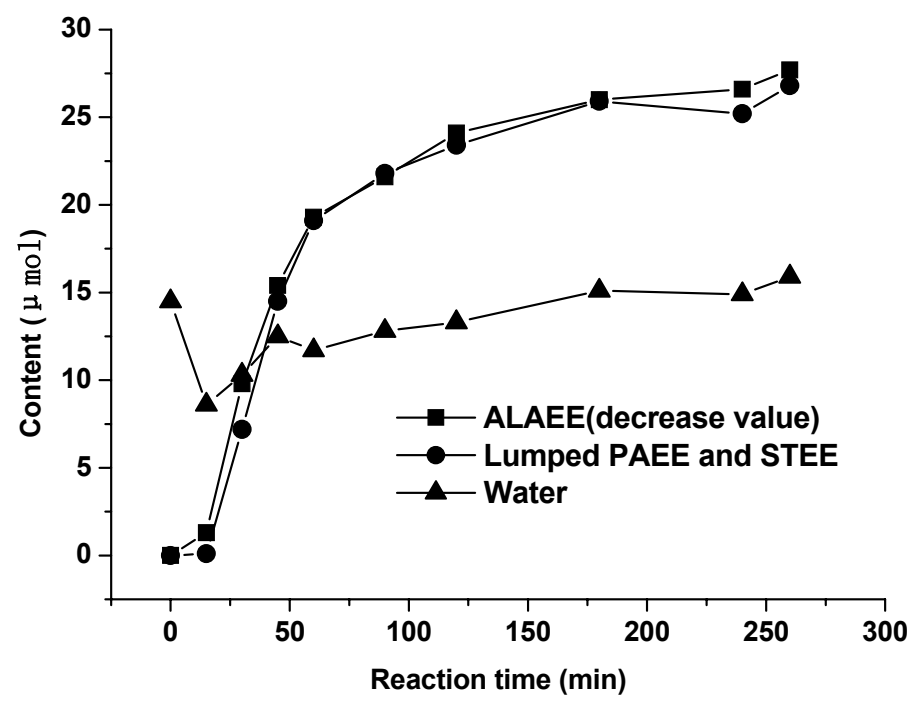

Figure 4. Contents of the constituents in the solution as a function of. reaction time. Reaction condition: PC (100.1 mg), ALAEE (140.0 mg), lipase (100 mg), water content (5.0\%) 


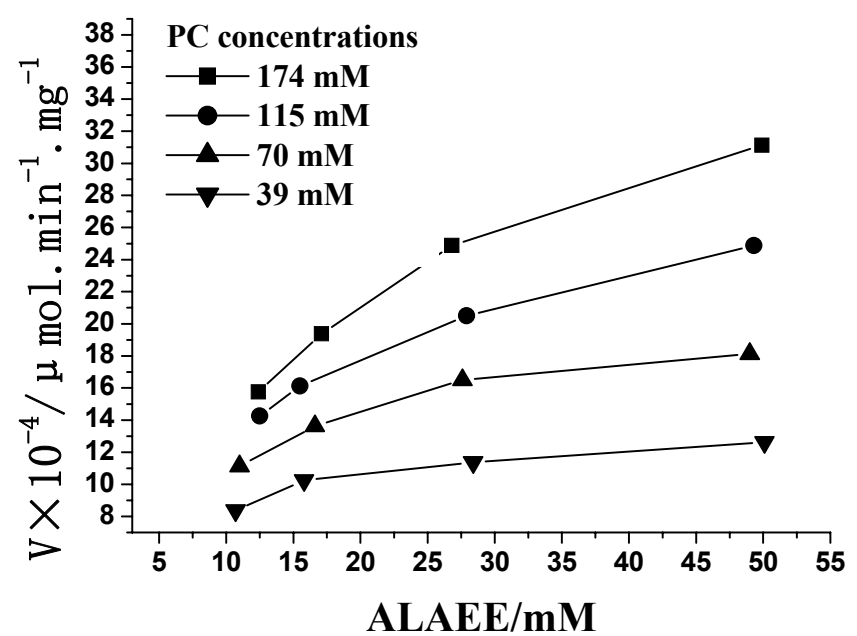

Figure 5. Effect of substrate concentration on the initial reaction

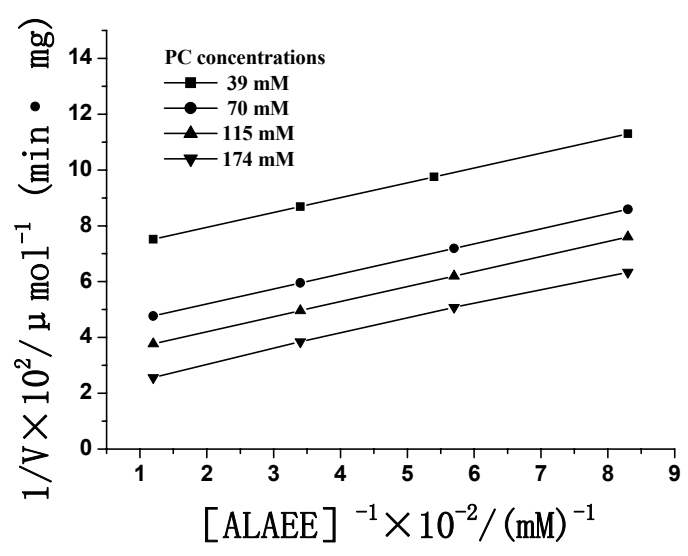

Figure 6. The plot of reciprocal initial velocity of the reaction versus reciprocal ALA ethyl ester concentration 


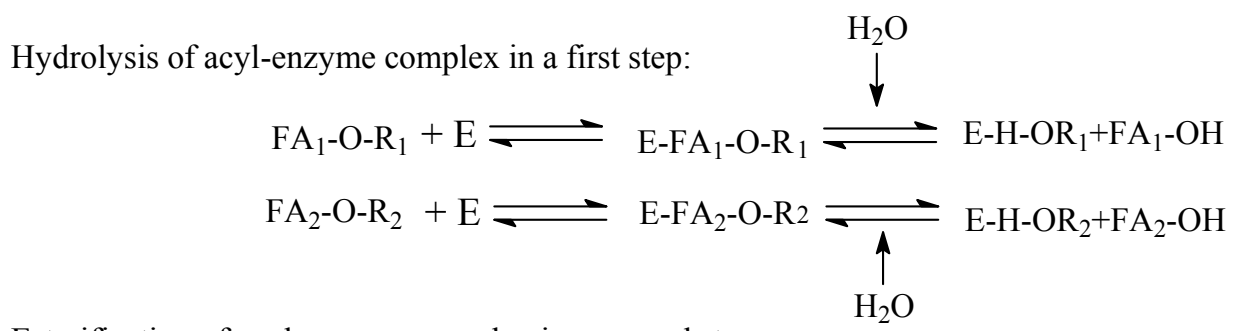

Esterification of acyl-enzyme complex in a second step:

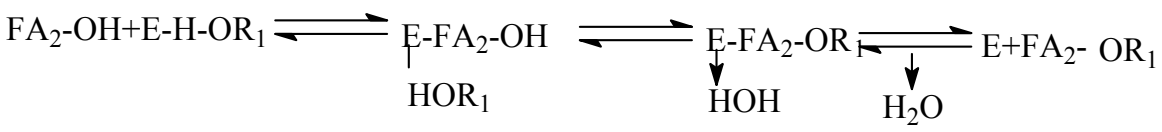

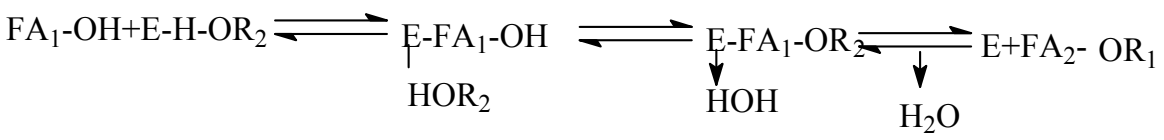

Mechanism of Ping-Ping Bi-Bi for the two-step transesterification:
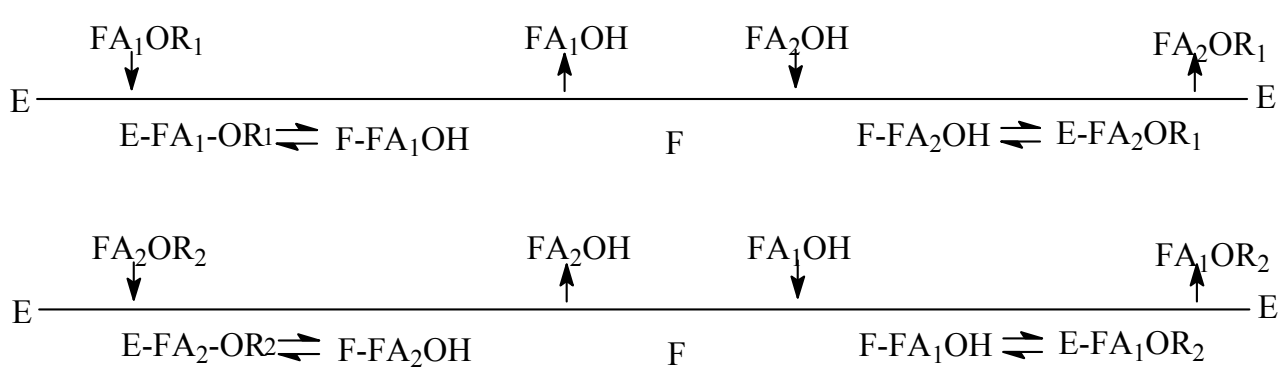

Figure 7. The scheme of the reaction steps $\left(\mathrm{R}_{1}\right.$ and $\mathrm{R}_{2}$ represent phospholipid glycerol, and ethanol, respectively)

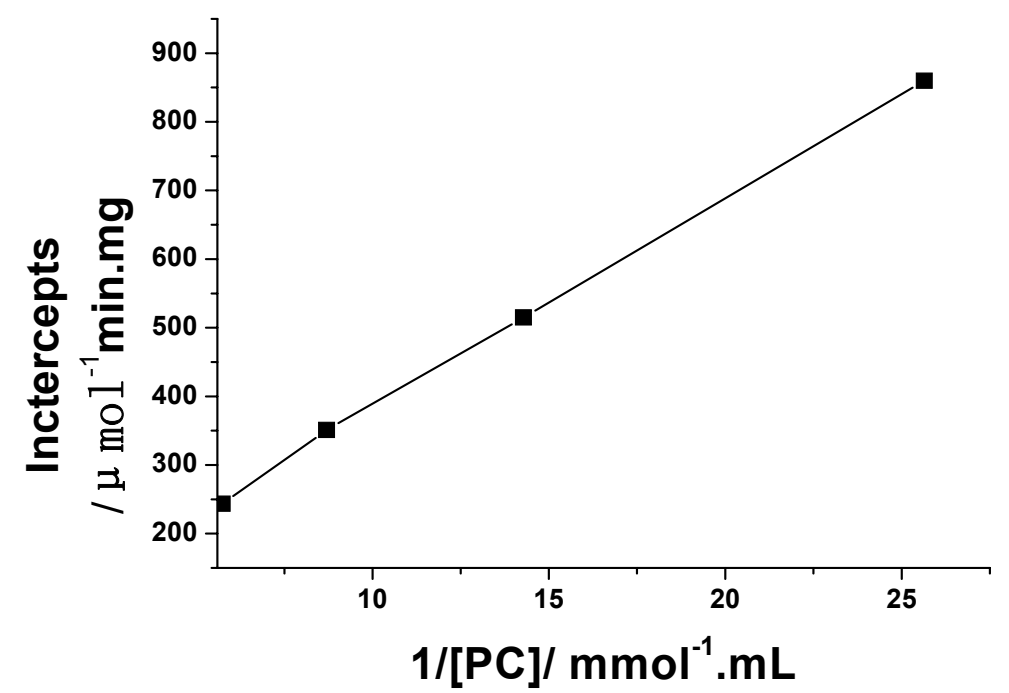

Figure 8. Intercepts of the straight lines in the primary plot in Fig. 3 versus the reciprocal PC concentrations 


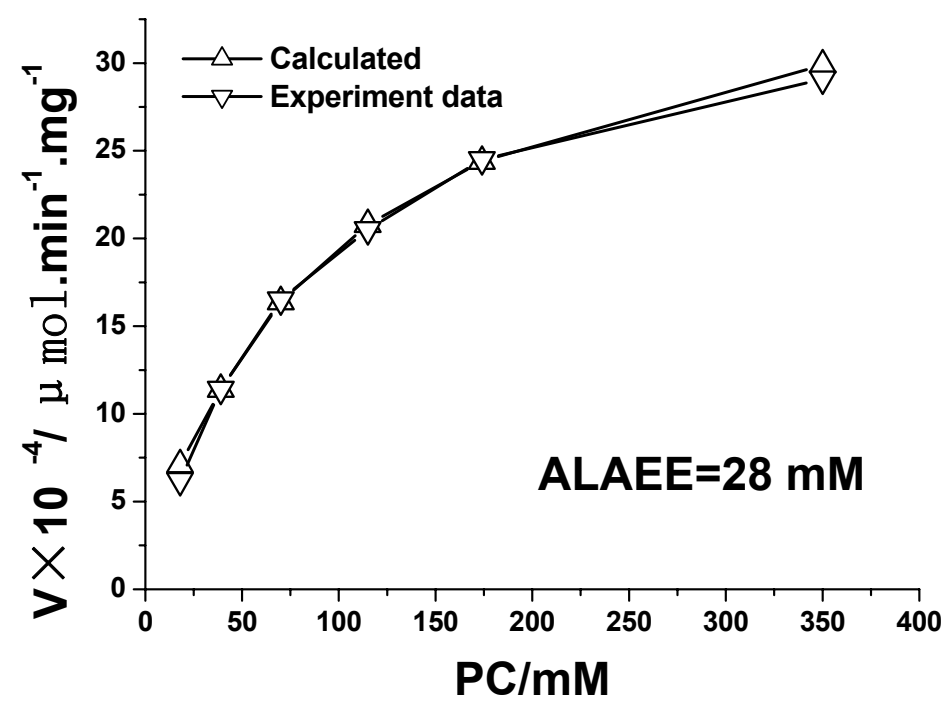

Figure 9. Comparison of experimental rate data and the rate data calculated by the kinetic model 